\title{
Trends in inpatient care for psychiatric disorders in NHS hospitals across England, 1998/99-2019/20: an observational time series analysis
}

\author{
Michelle Degli Esposti ${ }^{1}$ [ Hisham Ziauddeen ${ }^{2} \cdot$ Lucy Bowes $^{3} \cdot$ Aaron Reeves $^{1} \cdot$ Adam M. Chekroud $^{4}$. \\ David K. Humphreys ${ }^{1} \cdot$ Tamsin Ford $^{2}$
}

Received: 19 July 2021 / Accepted: 5 December 2021 / Published online: 24 December 2021

(c) The Author(s) 2021

\begin{abstract}
Purpose It is unclear how hospitals are responding to the mental health needs of the population in England, against a backdrop of diminishing resources. We aimed to document patterns in hospital activity by psychiatric disorder and how these have changed over the last 22 years.

Methods In this observational time series analysis, we used routinely collected data on all NHS hospitals in England from 1998/99 to 2019/20. Trends in hospital admissions and bed days for psychiatric disorders were smoothed using negative binomial regression models with year as the exposure and rates (per 1000 person-years) as the outcome. When linear trends were not appropriate, we fitted segmented negative binomial regression models with one change-point. We stratified by gender and age group [children ( $0-14$ years); adults $(15$ years +$)$ ].

Results Hospital admission rates and bed days for all psychiatric disorders decreased by 28.4 and $38.3 \%$, respectively. Trends were not uniform across psychiatric disorders or age groups. Admission rates mainly decreased over time, except for anxiety and eating disorders which doubled over the 22-year period, significantly increasing by $2.9 \%$ (AAPC $=2.88 ; 95 \%$ CI: $2.61-3.16 ; p<0.001)$ and $3.4 \%(\mathrm{AAPC}=3.44 ; 95 \% \mathrm{CI}: 3.04-3.85 ; p<0.001)$ each year. Inpatient hospital activity among children showed more increasing and pronounced trends than adults, including an increase of $212.9 \%$ for depression, despite a $63.8 \%$ reduction for adults with depression during the same period.

Conclusion In the last 22 years, there have been overall reductions in hospital activity for psychiatric disorders. However, some disorders showed pronounced increases, pointing to areas of growing need for inpatient psychiatric care, especially among children.
\end{abstract}

Keywords Psychiatry $\cdot$ Mental health $\cdot$ Hospital admissions $\cdot$ Time trends $\cdot$ Hospital episode statistics

\section{Introduction}

Michelle Degli Esposti

mdesposti@gmail.com

1 Department of Social Policy and Intervention, University of Oxford, Barnett House, 32 Wellington Square, Oxford OX1 2ER, UK

2 Department of Psychiatry, University of Cambridge, Cambridge CB2 3EB, UK

3 Department of Experimental Psychology, University of Oxford, Oxford OX2 6GG, UK

4 Department of Psychiatry, Yale University School of Medicine, New Haven, CT 06510, USA
Health services are facing unprecedented financial and operational pressures [1]. With England's expanding and ageing population, demand for services is outpacing the rate at which the NHS's budget is growing [2,3]. A policy objective aimed at meeting this challenge is to develop communitybased alternatives to reduce costly inpatient hospital care. Mental health services, in particular, have been transformed to shift the balance away from hospital and towards community care [4]. In the last 30 years, the number of mental health beds has been reduced by $73 \%$ [5]. These reductions have released funds in the short term, enabling the locus of psychiatric care to be moved outside the hospital. Although community-based models of care promise early and more 
sustainable intervention [4], there is now growing evidence that there are too few mental health beds to meet psychiatric need [6-8]. This raises important questions around how shrinking hospital provision impacts on the management of mental health conditions.

With 1 in 4 experiencing a mental health and/or substance use disorder at some point during their lives [9], mental illness has become the single largest cause of disability in England - costing the NHS over $£ 13.3$ billion in 2019/20 alone [10] — and is on the rise among both children and adults [11-13]. Repeated population-based surveys find that the prevalence of common mental disorders has increased by 2\% from 1999 to 2017 in children and by $2 \%$ from 1993 to 2014 in adults $[2,14]$. Despite this evidence of deterioration in mental health, it is not possible to draw a direct line from this general rise in poor mental health to an increased need for acute psychiatric care as only $0.2 \%$ of people with poor mental health and $1.8 \%$ of people with severe mental health disorders receive inpatient care among adults [2]. Inpatient admission for children's mental health is even less common.

However, measures of hospital activity for mental health point towards an increasing need for acute psychiatric care, despite diminishing resources. Since 1998/99 occupancy rates of mental health beds have exceeded the recommended $85 \%$ threshold for delivering high quality and safe care $[8$,
15]. Occupancy rates have risen even further in the last 10 years, resulting in patients having to be admitted out-ofarea, often at considerable distance from home, because of a lack of local beds $[16,17]$. In addition, A\&E activity for psychiatric conditions more than doubled between 2009/10 and 2019/20, further suggesting an increasing need for acute psychiatric care [18]. Taken together, this suggests a divergence between need and provision as hospital activity for psychiatric conditions increases, while resources for inpatient care are reduced (see Fig. 1).

Those receiving inpatient care seem to be more unwell. Based on scores on the Health of the Nation Outcome Scales (HoNOS), a widely used measure of health, impairment, and social functioning in adults with mental illness, adults admitted to inpatient care in 2018 were found to be more severely unwell (higher average HoNOS scores), than those admitted in 2013. Similarly, at discharge, average HoNOS scores at the point of discharge have been higher in recent years [8]. These comparisons indicate that the threshold for admitting people with severe mental illness has increased, while thresholds for discharging patients have fallen. In addition, compulsory detentions under the Mental Health Act have more than doubled over the last 30 years $[19,20]$. It is unclear to what extent this is because improved community services are able to look after people with severe

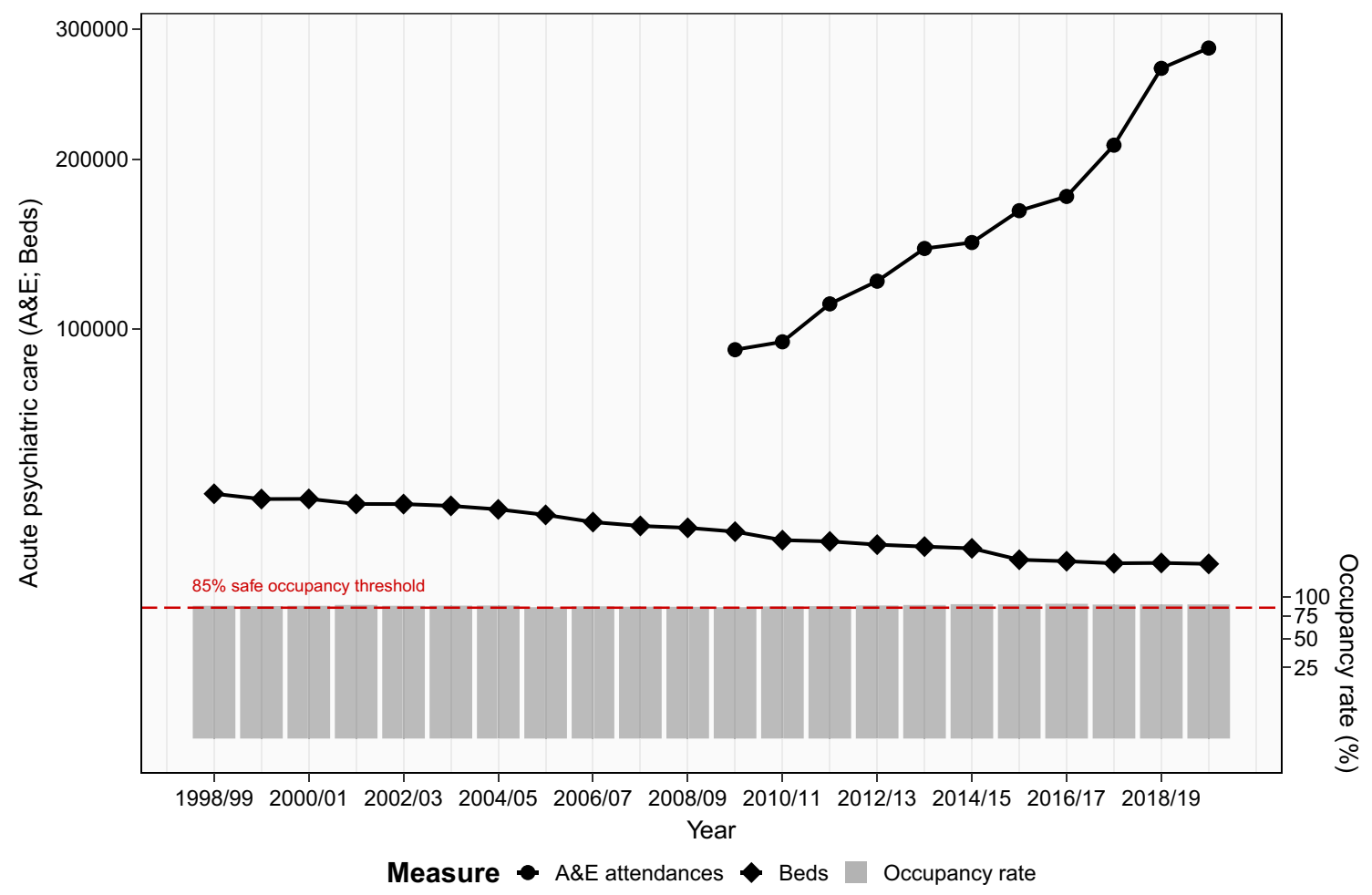

Fig. 1 Measures of acute psychiatric provision from 1998/99 to 2019/20, including counts of A\&E attendances for psychiatric conditions (circle scatter plot), mental health beds (diamond scatter plot), and rate of mental health bed occupancy (\%, grey bar chart). Where A\&E attendances and occupancy rates indicate need, while beds represent resources. Y-axes are plotted on a square-root scale 
mental illness in the community with only the most severely ill needing hospital admission, or because the shortage of mental health beds means they have to be prioritised for the most unwell [20-22]. Nevertheless, the fact that compulsory detentions make up a higher proportion of mental health bed occupancy reflects the severity of illness in those receiving hospital care.

While reductions in hospital provision are well documented, it is less clear how the remaining resources are being allocated in a system under strain. In this study, we use Hospital Episode Statistics (HES), which are routinely collected data from all hospitals across England [23], to explore how inpatient care (hospital admissions and bed days) is being used to meet acute psychiatric need, and if and how this has changed over the last 22 years as need increases and hospital provision continues to diminish.

\section{Method}

This observational time series analysis used routinely collected data on inpatient hospital activity in England from $1998 / 99$ to 2019/20. Ethical approval was not required, because all data were fully anonymised, pre-published, and publicly available. In line with best-reporting practices, we followed general recommendations set-out in the REporting of studies Conducted using Observational Routinely collected health Data (RECORD) statement [24].

\section{Data source}

The Department of Health routinely collects data on all admissions to NHS hospitals in England and independent sector providers (private or charitable hospitals) commissioned by the NHS: Hospital Episode Statistics Admitted Patient Care (HES APC) [23]. HES APC offers the opportunity to estimate population-based hospital admissions for psychiatric disorders as it measures all NHS hospital admissions. A hospital admission covers any secondary care-based activity that requires a hospital bed, including both emergency and planned admissions and day cases, but not including accident and emergency (A\&E, emergency department) attendances, or outpatient bookings. HES data, therefore, include hospital admissions that have been made following contact with specialist mental health services, as well as admissions after attending A\&E. We extracted the primary diagnoses for each HES APC entry, which represents the main reason the patient is receiving hospital care. Diagnoses were coded using the International Classification of Diseases version 10 (ICD-10). The HES APC database is collated and curated by NHS Digital.

\section{Study data}

We obtained HES data for each financial year (1st April to 31st March) from 1998/99 to 2019/20. These dates were chosen as it was the longest available time period with consistent ICD codes (i.e., version ICD-10). We used ICD-10 codes for common mental, behavioural, and neurodevelopmental disorders (see Supplementary Table 1 for full details) for primary diagnoses (3-character) to consistently identify hospital admissions and bed days over time. Outcome measures were aggregated for all common psychiatric disorders (ICD-10 codes: F00- F91) and broken down by specific disorder type (e.g., depression, see Supplementary Table 1).

\section{Main outcomes}

Our main outcomes were hospital admissions and overnight bed use, measured by bed days and length of stay. For hospital admissions and episodes, we modelled age-specific rates per 1000 person-years to control for changes in population size and by age group. We obtained age-specific population estimates for England from the Office for National Statistics' (ONS) mid-year population estimates.

\section{Admissions}

Rates of admissions (or finished admission episodes) per 1000 person-years of the total population. Hospital admissions represent the first period of inpatient care under one consultant within one healthcare provider. Admissions are counted against the year in which they end, and do not represent the number of patients, as a person may have more than one admission within the year [23]. Because admissions measure the first period of care, they are less prone to double counting from changes to the provision of inpatient care. As a result, they represent a subset of all hospital episodesaround 5\% of admissions have more than one episode of inpatient care.

\section{Bed days and length of stay}

Total bed days represent the sum of hospital episode duration, from admission to discharge date, for all episodes ending in the year. Measures of total bed days changed over the study period: from $1998 / 99$ to $2007 / 08$, bed days included estimates of unfinished episodes where finished episodes were uplifted by a factor to capture episodes that were not finished at the end of the year, whereas from 2008/09 onwards, total bed days only captured finished episodes for that year and were not uplifted by any estimation factor. 
Because any observed changes in bed days from 2008/09 may be confounded by this measurement change, we supplemented bed days with measures of length of stay. Information on length of stay was only available as summary statistics (mean or median) aggregated for each diagnosis for each year. To guard against skew from outliers, we extracted the median length of hospital spell duration in days instead of the mean. A spell is a period of continuous admitted patient care within a particular provider, calculated by subtracting the admission date from the discharge date for finished episodes. Day cases, which have a length of stay of zero bed days, were excluded from both estimates of total bed days and median length of stay.

\section{Secondary outcomes}

To characterise patients being admitted for psychiatric hospital care, we obtained information on finished consultant episodes broken down by gender and age.

\section{Episodes by gender and age}

Rates of episodes (or finished admission episodes) per 1000 person-years of the age-specific population. Finished consultant episodes (FCE) represent a continuous period of admitted patient care under one consultant within one healthcare provider. FCEs are counted against the year in which they end and are more prone to double counting as a person may have more than one episode of care within the same stay in hospital or in different stays in the same year [23]. Admissions represent a subset of FCEs as they measure only the first FCE and are therefore less susceptible to double counting (see Supplementary Fig. 1). Unlike admissions however, information on gender and age were publicly available for FCEs for our study period. We extracted demographic information on gender (male, female, and unspecified gender) and age $(0-14 y ; 15 y+)$. These gender and age categories were used as only information on males (i.e., not on females) and broader age groups (age 0-14y; 15-59y; $60-74 y ; 75 y+)$ were collected consistently over time.

\section{Statistical analysis}

We analysed trends in the yearly hospital admissions for psychiatric disorders by fitting negative binomial regression models with year as the exposure and the number of admissions as the main outcome, as there was significant evidence $(p<0.05)$ of overdispersion [25]. Year and age-specific population estimates (log-transformed) were included as an offset variable to adjust for changing population size and age structure. To handle any non-linearity between year and psychiatric hospital episodes, and more accurately model rate of change over the study period, we conducted change-point analyses. Change-point analysis is a data-driven, exploratory method that checks whether the outcomes follow a consistent linear trend over time or may be better explained by two trends-allowing for one change in slope gradient and/or trend direction [26]. For our change-point analyses, we compared simple linear (continuous) models against segmented regression models with two slopes, including a parameter for the time at which the change of slopes occurred (i.e., the change-point). Changepoints were estimated by iteratively fitting the linear model with a linear predictor through the segmented package in $\mathrm{R}$ (version 3.5.2) [27]. We selected the best-fitting model based on Akaike's information criterion (AIC) - low AIC values were preferred-and performing the (pseudo) score statistics to evaluate whether incorporating a change-point improved model fit $(p<0.05)$ [28].

We examined both annual percentage and overall changes to interpret trends. We calculated the average annual percentage changes (AAPCs) and $p$ values from our bestfitting regression models (i.e., simple or segmented, see above). AAPCs represent whether trends linearly increase or decrease from 1998/99 to 2019/20 while accounting for the uncertainty in the detected change points for segmented models [28, 29]. APPCs further estimate the average amount of percentage change for each year and indicate whether these year-on-year changes are significant. We supplemented these measures by calculating overall absolute change and relative change (i.e., percentage change) for the earliest year (1998/99) and most recent (2019/20) available year, as well for the estimated change-point and most recent year $(2019 / 20)$ for segmented models. To visualise trends, we plotted estimated counts from the best-fitting models (simple or segmented) and thus most accurately modelled changes over time.

\section{Results}

Table 1 describes inpatient care for psychiatric disorders from 1998/99 to 2019/20. Alcohol use disorder resulted in the highest number of hospital admissions and episodes (Supplementary Fig. 1), accounting for $26.9 \%$ of adult and $47.6 \%$ of child inpatient episodes. However, alcohol use disorder only accounted for $4.5 \%$ of bed days as it had the second shortest hospital stay. Schizophrenia had the second highest number of admissions and accounted for almost half of all bed days (42.7\%), with the length of hospital stay only exceeded by dementia and bipolar disorder (Supplementary Fig. 2). Eating disorders (17.8\%) and anxiety disorders (10.8\%) were the next most common indication for children's inpatient care. There was little difference between males and 
Table 1 Descriptive statistics for hospital admissions and bed days for psychiatric disorders, 1998/99-2019/20

\begin{tabular}{|c|c|c|c|c|c|c|c|c|}
\hline & \multirow{3}{*}{$\begin{array}{l}\text { Hospital } \\
\text { admissions } \\
\text { (count) }\end{array}$} & \multirow{3}{*}{$\begin{array}{l}\text { Total bed days } \\
\text { (count) }\end{array}$} & \multirow{3}{*}{$\begin{array}{l}\text { Median length of } \\
\text { stay (mean (SD)) }\end{array}$} & \multicolumn{5}{|l|}{ FCEs } \\
\hline & & & & \multirow[t]{2}{*}{ Total (count) } & \multicolumn{2}{|c|}{ Gender (all ages) } & \multicolumn{2}{|c|}{ Age (all genders) } \\
\hline & & & & & Male $(\%)$ & $\begin{array}{l}\text { Female \& } \\
\text { unknown } \\
(\%)\end{array}$ & Child (\%) & Adult (\%) \\
\hline $\begin{array}{l}\text { Common psychiatric } \\
\text { disorders }\end{array}$ & $3,333,418$ & $123,531,860$ & $14.8(1.66)$ & $4,267,571$ & 53.65 & 46.35 & 1.5 & 98.08 \\
\hline $\begin{array}{l}\text { Alcohol use dis- } \\
\text { order }\end{array}$ & 865,942 & $5,522,892$ & $2.23(1.34)$ & $1,160,429$ & 69.96 & 30.04 & 2.62 & 96.84 \\
\hline Anxiety & 184,249 & $2,401,566$ & $5.6(3.26)$ & 214,091 & 36.46 & 63.54 & 3.21 & 96.52 \\
\hline Bipolar disorder & 231,975 & $12,181,797$ & $30.68(2.32)$ & 289,348 & 40.99 & 59.01 & 0.11 & 99.53 \\
\hline Conduct disorders & 7037 & 116,259 & $1.91(1.02)$ & 7651 & 60.68 & 39.32 & 50.86 & $48.88^{\dagger}$ \\
\hline Dementia & 359,665 & $19,012,144$ & $33.14(7.07)$ & 551,242 & 41.68 & 58.32 & 0.01 & 99.78 \\
\hline Depression & 506,585 & $18,038,321$ & $20.39(3.07)$ & 580,579 & 40.31 & 59.69 & 0.77 & 98.95 \\
\hline Eating disorders & 48,564 & $2,106,319$ & $15.00(3.77)$ & 62,840 & 9.30 & 90.70 & 18.04 & 81.73 \\
\hline OCD & 12,756 & 626,580 & $20.43(2.75)$ & 14,305 & 52.51 & 47.49 & 6.81 & 92.94 \\
\hline $\begin{array}{l}\text { Personality disor- } \\
\text { ders }\end{array}$ & 190,238 & $6,198,083$ & $10.36(1.11)$ & 224,492 & 32.39 & 67.61 & 0.13 & 99.33 \\
\hline PTSD & 109,437 & $1,931,199$ & $7.82(0.66)$ & 122,050 & 52.14 & 47.86 & 1.08 & 98.52 \\
\hline Schizophrenia & 642,799 & $52,738,338$ & $25.26(2.49)$ & 839,449 & 61.8 & 38.20 & 0.25 & 99.35 \\
\hline $\begin{array}{l}\text { Substance use } \\
\text { disorders }\end{array}$ & 174,171 & $2,658,363$ & 4.75 (1.78) & 201,095 & 71.73 & 28.27 & 0.94 & 98.01 \\
\hline $\begin{array}{l}\text { Population estimates } \\
\text { (per million) }\end{array}$ & 1148.66 & - & - & 1148.66 & 564.19 & 584.47 & 208.52 & 940.14 \\
\hline
\end{tabular}

FCEs finished consultant episodes, $O C D$ obsessive-compulsive disorder, $P T S D$ post-traumatic stress disorder, $S D$ standard deviation

Child includes individuals aged $0-14$ years and adult includes individuals ages 15 years and older; percentages for age groups do not add up to $100 \%$ due to missing information

$\$$ Represents the summed total of the 12 most common psychiatric disorders (see Supplementary Table 1 for full diagnostic details)

${ }^{\dagger}$ Because adults represent ages $15 \mathrm{y}+$, conduct disorders in this age group reflect conduct disorders among adolescents

females across all disorders (Supplementary Fig. 3), though expected differences were seen within disorders (Table 1 \& Supplementary Fig. 4). For example, alcohol and substance use disorders were more common among males (70-72\%), whereas personality and eating disorders were more common among females (67-91\%). The overall percentage of males admitted to hospital increased from approximately $50 \%$ in $1998 / 99$ to $55 \%$ in 2019/20 (see Supplementary Fig. 3).

Three-year moving averages identified that the number of hospital admissions for all psychiatric disorders decreased by over $18.1 \%$ across the 22 -year period (see Fig. 2). The equivalent of around 5000 fewer yearly hospital admissions for psychiatric disorders, even though England's population size increased by around $13.8 \%$ during this period (Supplementary Table 2). Modelling trends in admission rates while adjusting for changing population size identified a larger overall reduction of $28.4 \%$ (Table 2). Bed days also decreased over this period, with a reduction of $26.4 \%$ from $1998 / 99$ to $2019 / 20$ (Fig. 2 \& Supplementary Table 3). A sensitivity check using median length of stay mirrored the same decreasing trend as total bed days (Supplementary Fig. 5).

\section{Trends in hospital admissions by psychiatric disorders}

Trends in rates of hospital admissions varied by psychiatric disorder (Table 2; Fig. 3). Admission rates for seven out of the 12 psychiatric disorders showed overall reductions from 1998/99 to 2019/20: bipolar disorder, conduct disorders, dementia, depression, OCD, PTSD, and schizophrenia. Although the magnitude of the rate of change was not consistent over time for bipolar disorder, conduct disorders and dementia, all seven disorders significantly decreased each year. The largest reductions of around $70 \%$ were seen for conduct disorders, dementia, and depression-equivalent to a year-on-year decrease of 5.6-7.1\% - while schizophrenia showed the smallest significant decrease of $35.9 \%$. Only two psychiatric disorders, anxiety and eating disorders, showed consistently increasing trends, with admission rates almost doubling between 1998/99 and 2019/20, translating to 


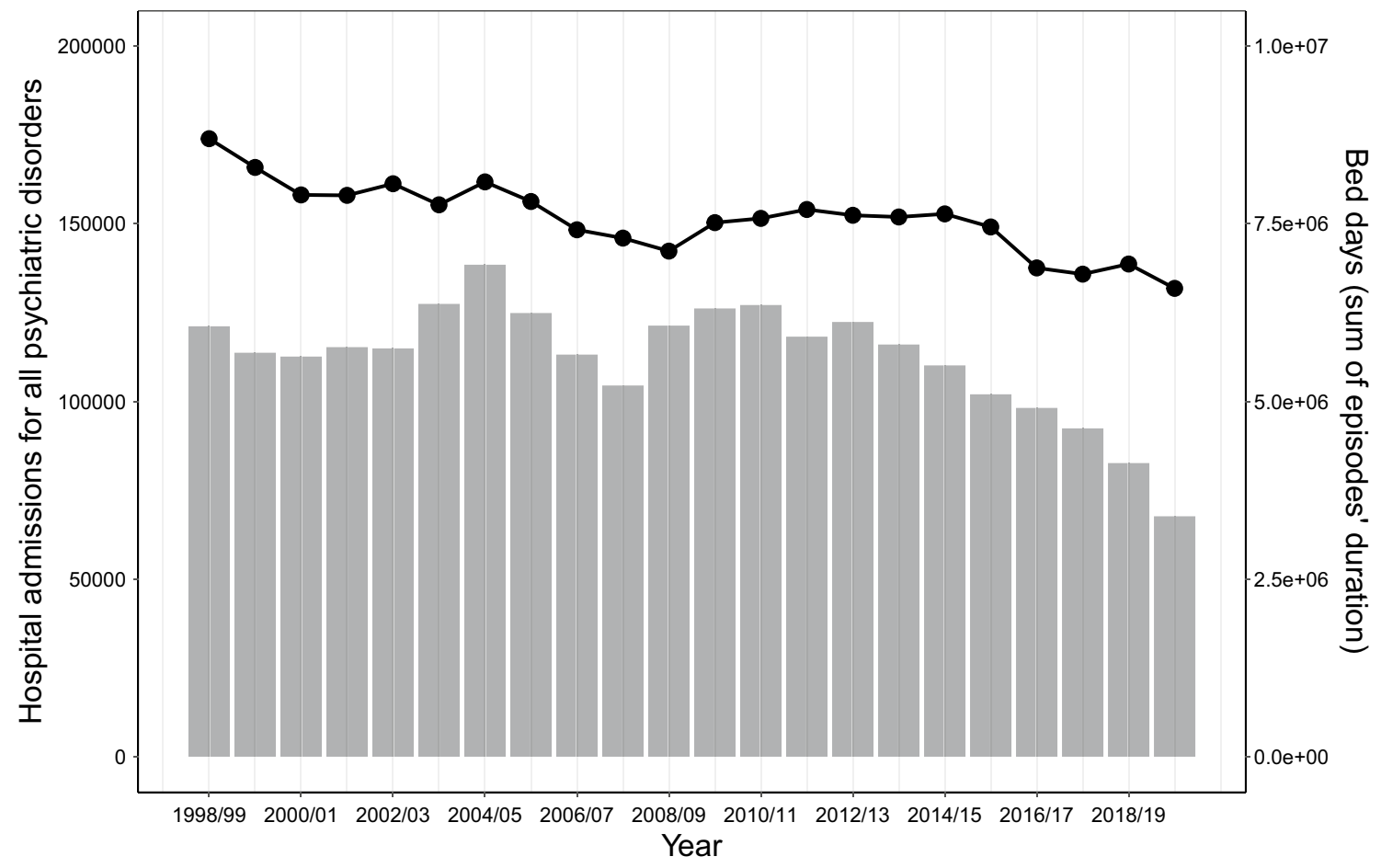

Fig. 2 Hospital admissions and bed days for all 12 common psychiatric disorders from 1998/99 to 2019/20

relative increases of 81.6 and $100.5 \%$ (respectively). These temporal patterns result in anxiety and eating disorders accounting for a larger proportion of all common psychiatric admissions in 2019/20 compared to 1998/99 (Supplementary Fig. 6).

Trends admission rates for three psychiatric disorders changed direction during the study period (Fig. 3). Admission rates for alcohol use disorder sharply increased from $1998 / 99$ to $2010 / 11$ and then shallowly decreased from 2010/11 onwards. Since the initial period of increase was greater than the following period of decrease, this resulted in an overall $32.1 \%$ increase. Substance use and personality disorders showed the opposite pattern: initially decreasing and then increasing. These changing trends meant that the average annual percentage changes for these disorders were small, ranging from $-0.8-0.6 \%$. However, when comparing from their estimated change points of 2008/09 for substance use disorders and 2006/07 for personality disorders, both showed notable increases of 28.4 and $44.8 \%$, respectively.

\section{Trends in hospital bed days by psychiatric disorders}

Trends in the total number of hospital bed days typically mirrored trends in admissions (see Fig. 4). Bed days for bipolar disorder, conduct disorders, dementia, depression, OCD, and PTSD all declined at a similar yearly rate to admissions. For example, from 1998/99 to 2019/20, admission rates for depression significantly decreased by $5.5 \%$ each year (AAPC: -5.55 ; $95 \%$ CIs: -5.77 to -5.33 ; $p<0.001$, while bed days also decreased by a similar magnitude of $5.3 \%$ each year (AAPC: -5.29 ; 95\% CIs: -5.52 to $-5.05 ; p<0.001)$. However, a diverging pattern was seen with some disorders. Despite a substantial $81.6 \%$ rise in rates of admissions for anxiety, bed days reduced by $29.6 \%$ over the same period. While admissions for eating disorders followed a steep and consistent rate of increase from 2003/04 onwards, bed days for eating disorders went from increasing to decreasing by $12.5 \%$ from $2015 / 16$ to $2019 / 20$. Admission rates for alcohol use disorder increased by $32.1 \%$ over the study period and by $28.4 \%$ for substance use disorders from 2008/09, but bed days for both conditions consistently decreased.

These discrepancies in the total number of bed days compared to admissions may be explained by changes in median length of hospital stay for each admission. Although median length of hospital stay was relatively stable over time, shortening of hospital stays was visible for alcohol use disorder, anxiety, conduct disorders, eating disorders, and substance use disorders (see Supplementary Fig. 7). For example, length of stay for anxiety reduced from 10.3 days down to 2.1 , shortening the typical length of hospital by around $80 \%$ (see Supplementary Table 4). 


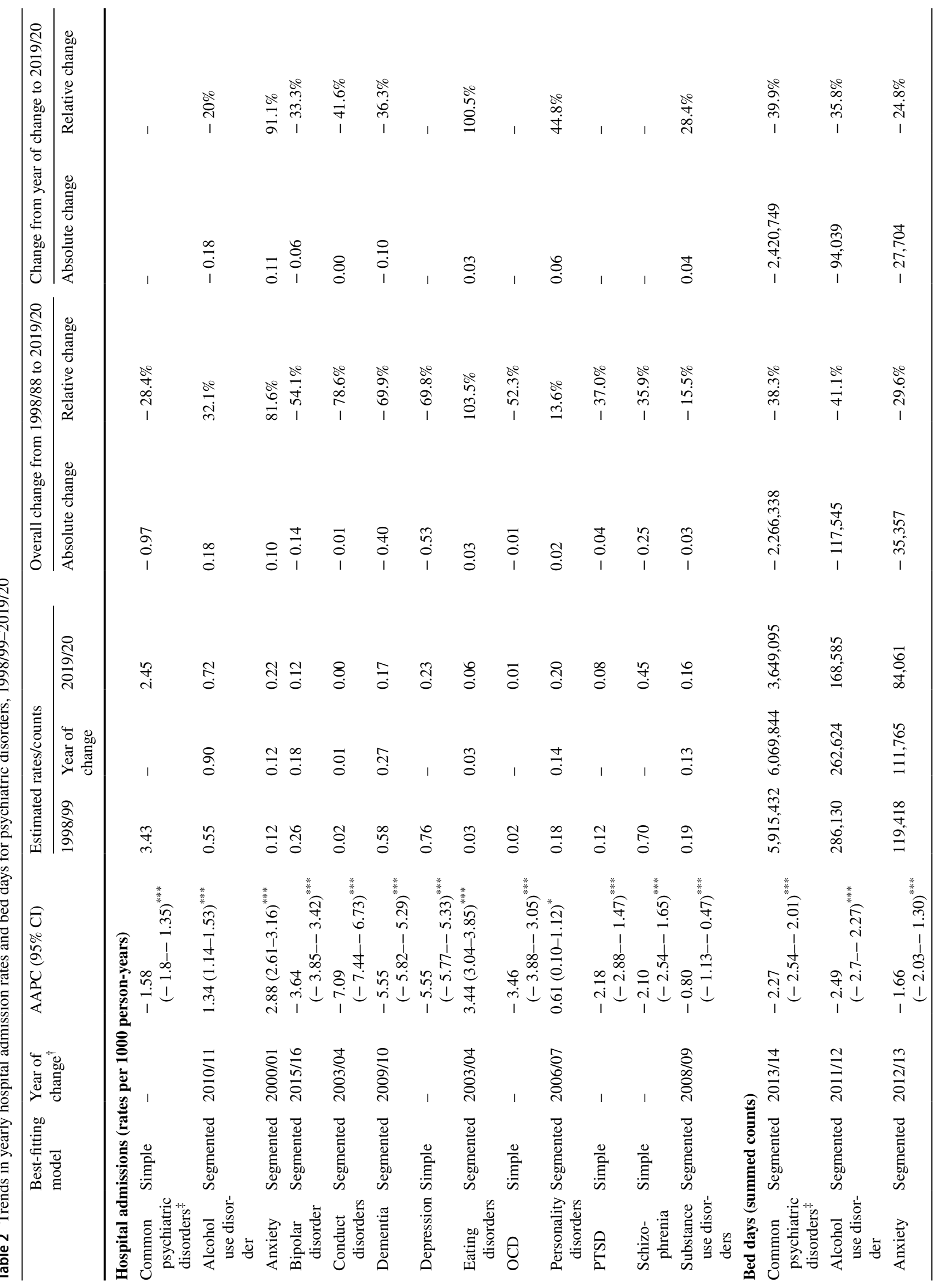




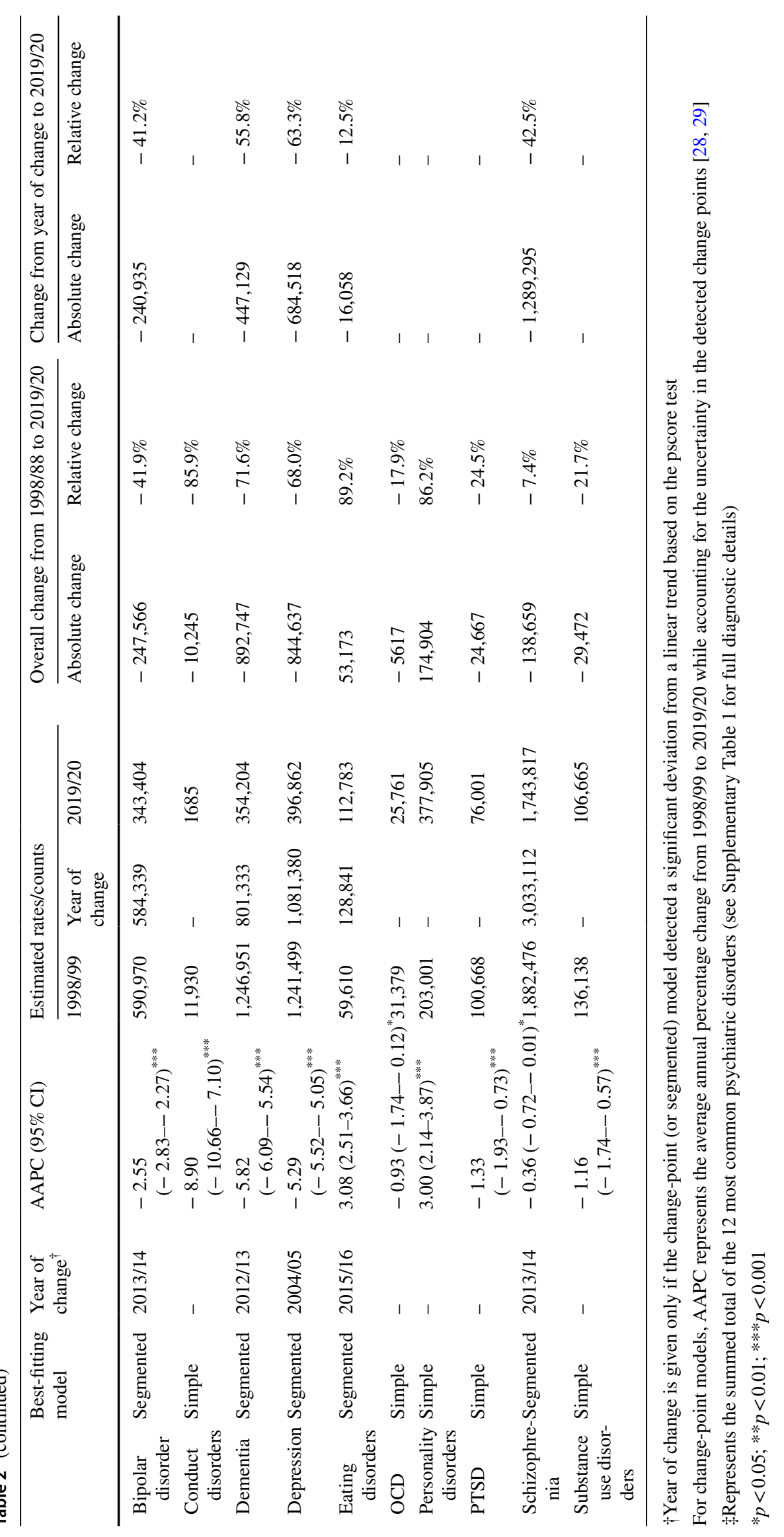




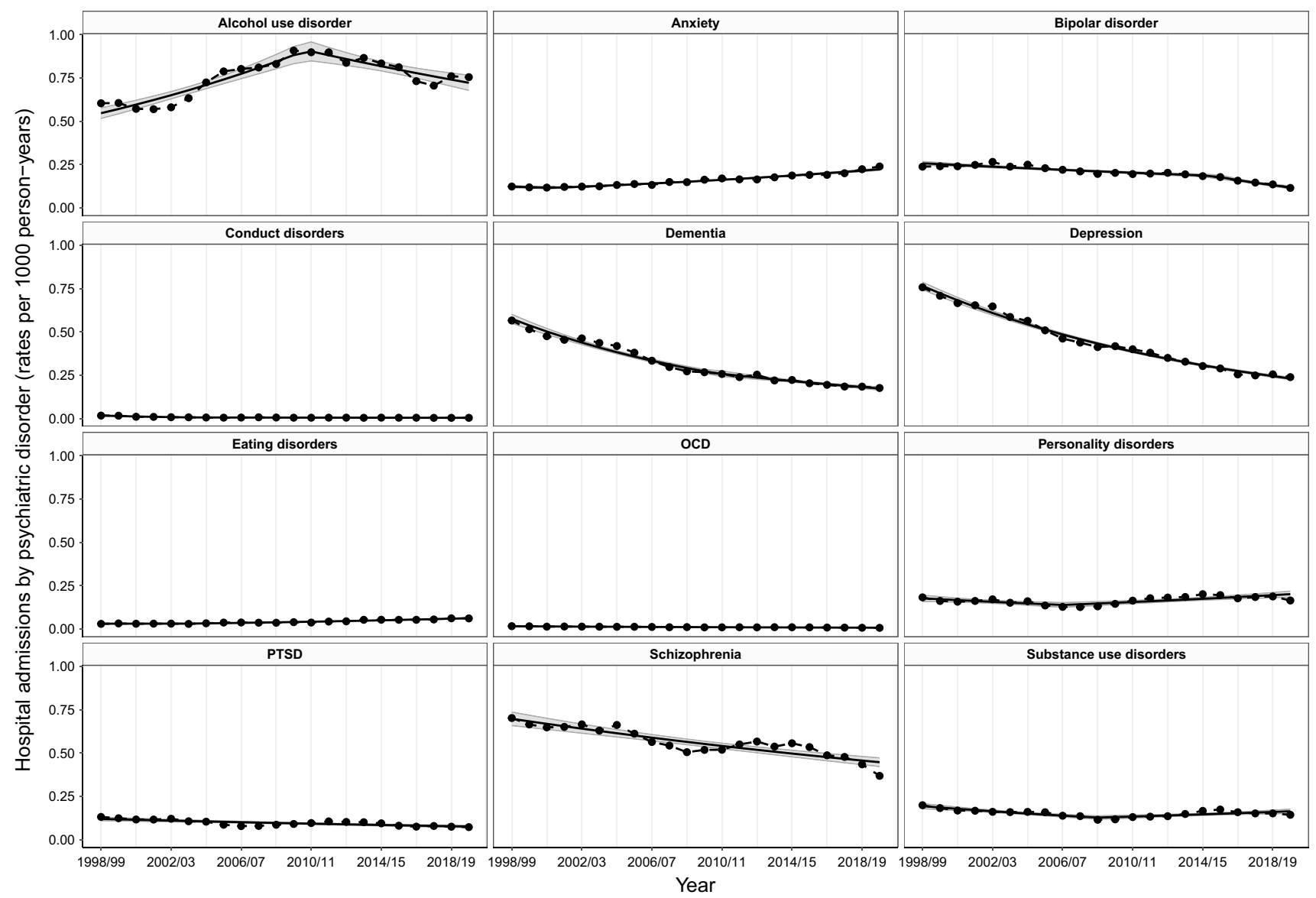

Fig. 3 Trends in hospital admission rates by psychiatric disorder, 1998/99-2019/20

\section{Trends in psychiatric hospital episodes by age group}

Rates of hospital episodes for psychiatric disorders disaggregated by adults and children are presented in Supplementary Table 5. Trends in rates for adults followed the same patterns as overall admission rates (Supplementary Fig. 8), since most hospital episodes were for adults (Table 2). However, trends for children showed marked differences, where there were pronounced increases in mental health disorders requiring hospital care compared to adults (Supplementary Fig. 9). For example, despite hospital activity for depression consistently declining by $4.7 \%$ each year among adults (AAPC: -4.72 ; $95 \%$ CIs: -5.00 to -4.43 ; $p<0.001$ ), it consistently increased by $5.6 \%$ among children (AAPC: 5.58 ; 95\% CIs: $4.48-6.69 ; p<0.001$ ). This meant that trends in depression showed stark diverging trends by age group, with large reductions in adults yet large upsurges in children. Although hospital care for anxiety and eating disorders increased for both age groups, the rise was more pronounced among children. Anxiety increased by $283.3 \%$ and eating disorders by $361.2 \%$ in children, compared to 98.1 and $167.6 \%$ (respectively) in adults. In addition, there was evidence of more hospital activity over time for OCD, PTSD, personality disorders, and schizophrenia for children compared to adults (see Supplementary Table 5).

\section{Discussion}

Using national data on hospital usage, we found that over the period $1998 / 99$ to $2019 / 20$, admissions for psychiatric disorders decreased by around $28 \%$ and the total number of bed days decreased by $38 \%$. These reductions in psychiatric hospital care were not uniform across mental health disorders or across age groups. Alcohol use disorder was the largest single reason for being admitted to hospitals for both children and adults, followed by schizophrenia and depression in adults, and by eating disorders and anxiety in children. For adults, admissions for most psychiatric disorders declined consistently, but those for anxiety and eating disorders doubled from 1998/99 to 2019/20. For children, on the other hand, admissions increased for several disorders. These changes in admissions over time were not always mirrored by changes to total number of bed days, reflecting 


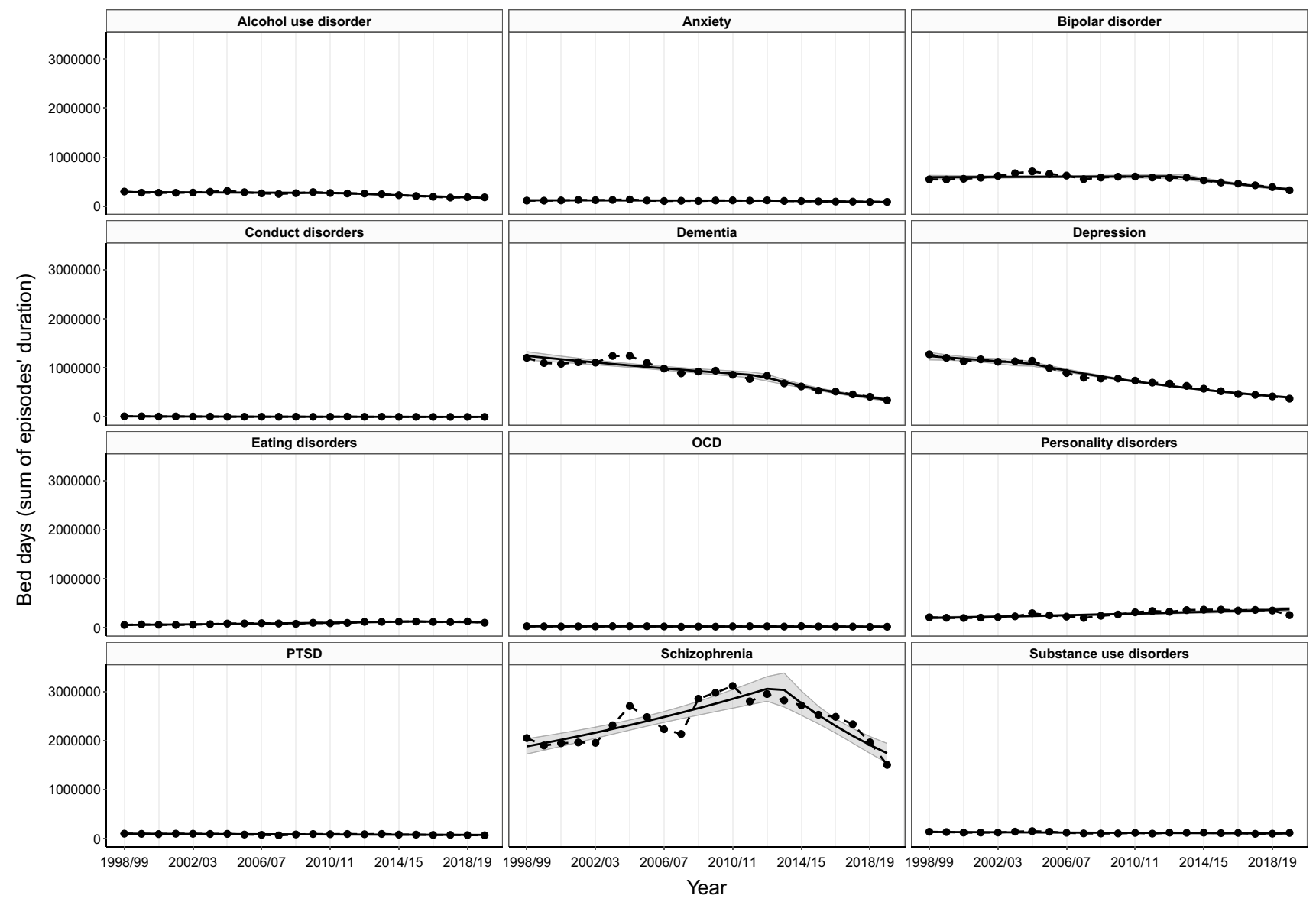

Fig. 4 Trends in hospital bed days (total counts) by psychiatric disorder, 1998/99-2019/20

the fact that the length of hospital stay for some disorders has been dramatically shortened in recent years. Our findings show that reductions in psychiatry hospital activity over the last 22 years have not occurred for all disorders or for children, highlighting areas of growing need for acute psychiatric care.

These findings are against the backdrop of long-term reductions in hospital provision and known shortages in mental health beds [5], as well as reductions in the resourcing of community mental health and social care during the study period. The HONOS data show that over time people are more unwell at admission and at discharge, pointing towards a growing pressure on inpatient resources. The known shortage in inpatient beds has previously been linked to subsequent increases in involuntary psychiatric hospital admissions, as the discrepancy between demand and supply may, in and of itself, exacerbate subsequent demand [21]. Cuts to community mental health and social care services that may have previously curtailed such demand for inpatient care by preventing hospital admissions could be further contributing to these changes [30]. An increased emphasis on community mental health provision through home treatment teams and assertive community outreach (ACT) may have helped to reduce inpatient care for severe mental illness since 2000 [31, 32]. However, it is important to bear in mind that home treatment teams were an integral part of bed reduction initiatives rather than a factor that led to a reduced need for beds, and many ACT teams across England have been decommissioned and disbanded in the last 10 years, leaving a potential demand for acute psychiatric care which hospitals are now under resourced to provide for.

A growing body of evidence shows that acute psychiatric care is a system under strain. The different trends in hospital admissions for psychiatric disorders may therefore reflect practical and operational decisions around service allocation. Patients with specific psychiatric disorders can be more easily allocated to general and acute hospital beds or specialist beds for specific diagnoses. For example, approximately $20 \%$ of hospital admissions are for patients with dementiamany of which go on to occupy general and acute hospital beds [33]. While the number of general and acute hospital beds has also reduced over time, these reductions have been less severe compared to the reductions in mental health beds [5]. In addition, multidisciplinary specialist inpatient 
dementia units (SIDU) have been developed within acute trusts to provide hospital care for dementia patients with more complex needs [34]. As a result, patients with psychiatric disorders that can be allocated to general acute hospital beds and dedicated specialist units, such as dementia, may be subject to less downward pressure than those requiring mental health beds specifically. The provision of inpatient psychiatric care for children and adolescents may also be subject to unique operational pressures. This is supported by figures showing an increasing number of children and adolescents being inappropriately placed in adult psychiatric wards, as well as inappropriate out-of-area placements, to receive acute psychiatric care [35, 36]. Again, however, the burden of supplying psychiatric beds to children and adolescents in need will vary by psychiatric disorders, since patients with some mental health problems (e.g., eating disorders) can more readily receive acute care in general paediatric wards compared to other mental health problems (e.g., schizophrenia) [37].

The changing trends in hospital admissions do not closely match changes in prevalence of psychiatric disorders. Although we identified increases in inpatient care for mood-related disorders which may reflect increases in their prevalence in the general population, the changes in hospital admissions exceed the documented rises in disorder prevalence in the population $[2,38]$. In the case of anxiety disorders, rates of hospital admissions almost doubled from 1998/99 to 2019/20, while the prevalence of anxiety as identified by population surveys from 1997 to 2017 only increased by $2 \%$ [2]. This suggests that other factors are contributing to these increases in admissions for anxiety and personality disorders, including a lack of-or at least reduction in-community services for these conditions. On the other hand, the $70 \%$ reduction in hospital admissions for both depression and dementia is unlikely to reflect a decrease in the population prevalence of these disorders in England, given the evidence from national surveys and projections from modelling studies [2, 39]. This suggests that acute psychiatric services may not be providing for all people living with these psychiatric disorders, either because these people are receiving adequate care elsewhere (e.g., in the community) or because these psychiatric disorders are considered less of a priority for inpatient care when there are bed shortages.

Wider societal and economic factors need to be considered as well. Public health initiatives, such as those restricting the availability, affordability, and marketing of alcohol, may be contributing to a decline in drinking, even at the extreme end of the spectrum [40]. However, it is possible that reductions in hospital admissions for alcohol use disorder reflect changes in the frequency and patterns of substance use in the population with other substances becoming more prominent, which may explain the increase in admission for substance use disorder at a similar to time to the decline in admissions for alcohol use disorder. Another important factor here may be the impact of the economic recession in 2008/10 on inpatient psychiatric activity [41]. The significant cuts to mental health and social care following the recession, coupled with heightened financial and job instability, may have contributed to and worsened poor mental health [42]. Such findings need further careful examination to establish the effects of economic downturns on rates of psychiatric hospital activity [43]. Given the COVID-19 pandemic and the pressing need for services to effectively anticipate and respond to changing demands [11, 44], understanding the relationship between economic instability and community and inpatient psychiatric activity at the national level should be a research priority.

Our results identified more increasing and pronounced trends in psychiatric hospital care for children [45]. The change in the population prevalence of these conditions is similar among both adults and children and so these trends suggest that children are disproportionately requiring inpatient psychiatric care. CAMHS services turn away 1 in 4 referrals (around 52,500 children each year) [46]. Untreated, these patients may later present in crisis and place additional demands on the acute hospital setting. Inpatient hospital activity for children with eating disorders showed particularly steep increases [31], despite evidence showing that hospital care is not the best first line option [47]. Specifically, research indicates that family treatment outside of the hospital setting and specialist community services may be more effective than general child and adolescent mental health services (CAMHS) for treating young people with eating disorders [48]. Admissions for depression and schizophrenia were also rising faster among children than adults. Both trends suggest a need for improved prevention programmes, lower acuity treatment options, and community care to reduce the demand for hospital care through effective early intervention for children specifically. Future research should focus on improving our understanding of the role of specialist community services in preventing costly admissions to hospitals.

\section{Study limitations}

This was a descriptive association study and did not formally examine the role of causal factors (e.g., 2008/10 economic recession) in driving trends and changes over time. This paper cannot speak to why we observed these temporal patterns in hospital admissions for psychiatric disorders. Instead, our modelling approach aimed to descriptively summarise trends over the last 22 years by fitting linear or segmented linear models with one 
change-point. As a result, the models for some psychiatric diagnoses showed superior fit to others. Notably, the simple linear models failed to capture substantial variation in rates of hospital admissions for PTSD and schizophrenia, which both dipped around 2006/07 to 2008/09 and then spiked between 2011/12 and 2014/15. HES routine data are an invaluable source, but routinely collected data have their limitations. The data used in this study are restricted to admitted patient care and will thus underestimate the true burden on hospitals as they do not capture all A\&E or day attendances. We extracted and restricted the analyses to psychiatric disorders which were coded as the primary diagnosis - the main reason for receiving hospital careto minimise double counting from secondary diagnoses and avoid confounding from changing recording practices around dual diagnoses. Although this makes for a more focused and cleaner time trends analysis, it does miss out on important information on potential co-morbidities. In addition, the disaggregated data were only available for the age groupings $(0-14 \mathrm{y}$ and $15 \mathrm{y}+)$ over the study period, which do not map on to NICE-recommended divisions and obscure information on adolescents. Unlike gender and age which are well completed, we were unable to analyse data on ethnicity due to inconsistent and incomplete recoding [23]. We were also unable to analyse differences in hospital providers (general vs psychiatric hospitals) or type of psychiatric admission (voluntary vs involuntary) as these data are not published in the HES APC database. Up-to-date statistics on inpatients detained under the Mental Health Act (1983) are not yet available (last reporting period 2015/16). Patterns in involuntary psychiatric admissions detained under the Mental Health Act may play a key role in driving overall trends as previous evidence shows significant rises in involuntary admissions since the 1980s, which has been linked to economic deprivation, and warrants further investigation [19, 21, 49]. Finally, changing recording practices and priorities may undermine the temporal consistency of the psychiatric diagnoses. For example, in January 2007, there was a move from secondary uses clinical information being stored and managed by the NHS-Wide Clearing Service (NWCS) to the Service Secondary Uses Submissions (SUS), with the aim to make more clinical information electronically available. There were also changes to how the total number of bed days were estimated in 2003 and 2008. Although this may have affected the temporal consistency of the data during our study period, we did not identify unanimous changes in any of these years across psychiatric disorders. This suggests that the patterns seen for psychiatric hospital activity cannot be fully attributed to these recording changes and our smoothing of the trends are likely to have (at least partially) controlled for such inconsistencies over time.

\section{Conclusions}

Admissions to hospitals are expensive, and current policy objectives and guidance focus on trying to maintain support for individuals with poor mental health outside of the hospital setting. However, the patterns of hospital admissions identified here, and how they have changed over the last 22 years, show that not all psychiatric disorders or age groups are experiencing reductions in hospital activity. We observed increases in hospital admissions for anxiety and eating disorders, especially among children. This raises critical questions around whether the large-scale reductions in hospital provision and mental health beds over the last 30 years, alongside the reductions in community mental services in recent years, have left people more vulnerable and more likely to present to hospitals in crisis-even though hospitals have diminishing resources to provide for these acute mental health needs.

Supplementary Information The online version contains supplementary material available at https://doi.org/10.1007/s00127-021-02215-5.

Author contributions MDE conceived the study and designed it with help from HZ, LB, AR, DKH, and TF. MDE collected the data and conducted the analyses. MDE wrote the first draft of the paper. All authors assisted in the interpretation of data and revising the paper and approved the final draft.

Funding This research received no specific grant from any funding agency, commercial, or not-for-profit sectors.

Availability of data and materials All data are freely and publicly available from NHS Digital. A dataset of aggregated yearly data on Admitted Patient Care (Hospital Episode Statistics) is also available on request from the corresponding author at mdesposti@gmail.com.

Code availability All statistical code used in the analyses has been deposited at the Open Science Framework (https://osf.io/gn65w/?view_ only $=4 \mathrm{~b} 3 \mathrm{cdfc} 9787 \mathrm{~d} 40 \mathrm{~d} 0 \mathrm{bcf} 9 \mathrm{a} 53 \mathrm{faa} 36471 \mathrm{c}$ ) for purposes of reproducing or extending the analysis.

\section{Declarations}

Conflict of interest Dr. Chekroud reports other from Spring Care Inc, other from Fitbit Inc, from UnitedHealthcare Inc, outside the submitted work. In addition, Dr. Chekroud has a patent submission for three major depressive disorder treatments pending to a) USPTO docket number Y0087.70116US00, b) USPTO. Provisional Appl. No. 62/491,660, and c) USPTO. Provisional Appl. No. 62/629,041, and has consulted for Fortress Biotech on antidepressant drug development. All other authors have no relevant financial or non-financial interests to disclose.

Ethical approval Ethical approval was not required, because all data were fully anonymised, pre-published, and publicly available.

Open Access This article is licensed under a Creative Commons Attribution 4.0 International License, which permits use, sharing, adaptation, distribution and reproduction in any medium or format, as long as you give appropriate credit to the original author(s) and the source, 
provide a link to the Creative Commons licence, and indicate if changes were made. The images or other third party material in this article are included in the article's Creative Commons licence, unless indicated otherwise in a credit line to the material. If material is not included in the article's Creative Commons licence and your intended use is not permitted by statutory regulation or exceeds the permitted use, you will need to obtain permission directly from the copyright holder. To view a copy of this licence, visit http://creativecommons.org/licenses/by/4.0/.

\section{References}

1. Maguire D, Dunn P, McKenna H (2016) How hospital activity in the NHS in England has changed over time. In: Kings Fund. www.kingsfund.org.uk/publications/hospital-activity-fundingchanges

2. McManus S, Bebbington P, Jenkins R, Brugha T (2016) Mental health and wellbeing in England: the Adult Psychiatric Morbidity Survey 2014. NHS Digital

3. The King's Fund (2016) Demand for hospital services outstripping NHS funding. www.kingsfund.org.uk/press/press-releases/ demand-hospital-services-outstripping-NHS-funding

4. Giburt H (2014) Service transformation: Lessons from mental health. Kings Fund 24

5. The King's Fund (2020) NHS hospital bed numbers: past, present, future. www.kingsfund.org.uk/publications/nhs-hospitalbed-numbers

6. Knapp M, Chisholm D, Astin J et al (1997) The cost consequences of changing the hospital-community balance: the mental health residential care study. Psychol Med 27:681-692. https://doi.org/10.1017/S0033291796004667

7. Knapp M, Beecham J, McDaid D et al (2011) The economic consequences of deinstitutionalisation of mental health services: lessons from a systematic review of European experience. Health Soc Care Community 19:113-125. https://doi.org/10. 1111/j.1365-2524.2010.00969.x

8. The Strategy Unit (2019) Exploring Mental Health Inpatient Capacity across Sustainability and Transformation Partnerships in England. www.strategyunitwm.nhs.uk/publications/explo ring-mental-health-inpatient-capacity

9. Bebbington PE, McManus S (2020) Revisiting the one in four: the prevalence of psychiatric disorder in the population of England 2000-2014. Br J Psychiatry 216:55-57

10. NHS England (2017) Mental Health Five Year Forward View Dashboard. www.england.nhs.uk/publication/mental-healthfive-year-forward-view-dashboard/

11. Ford T, John A, Gunnell D (2021) Mental health of children and young people during pandemic. BMJ 372:n614. https://doi.org/ 10.1136/bmj.n614

12. Newlove-Delgado T, McManus S, Sadler K et al (2021) Child mental health in England before and during the COVID-19 lockdown. Lancet Psychiatry. https://doi.org/10.1016/S22150366(20)30570-8

13. Pierce M, Hope H, Ford $\mathrm{T}$ et al (2020) Mental health before and during the COVID-19 pandemic: a longitudinal probability sample survey of the UK population. Lancet Psychiatry 7:883-892. https://doi.org/10.1016/S2215-0366(20)30308-4

14. Ford T, Vizard T, Sadler K et al (2020) Data resource profile: The Mental health of children and young people surveys (MHCYP). Int J Epidemiol 49:363-364. https://doi.org/10.1093/ije/dyz259

15. Perry J, Palmer L, Thompson P et al (2017) Standards for inpatient mental health services. Royal College of Psychiatrists

16. NHS Digital (2016) Out of area placements. digital.nhs. uk/data-and-information/clinical-audits-and-registries/ out-of-area-placements-oaps
17. NHS Digital (2021) Bed availability and occupancy dataOvernight. www.england.nhs.uk/statistics/statistical-workareas/bed-availability-and-occupancy/bed-data-overnight/

18. NHS Digital (2021) A\&E Attendances and Emergency Admissions. www.england.nhs.uk/statistics/statistical-work-areas/aewaiting-times-and-activity/

19. Bindman J, Tighe J, Thornicroft G, Leese M (2002) Poverty, poor services, and compulsory psychiatric admission in England. Soc Psychiatry Psychiatr Epidemiol 37:341-345. https:// doi.org/10.1007/s00127-002-0558-3

20. Sheridan Rains L, Weich S, Maddock C et al (2020) Understanding increasing rates of psychiatric hospital detentions in England: development and preliminary testing of an explanatory model. BJPsych Open 6:E88. https://doi.org/10.1192/bjo.2020. 64

21. Keown P, Weich S, Bhui KS, Scott J (2011) Association between provision of mental illness beds and rate of involuntary admissions in the NHS in England 1988-2008: ecological study. BMJ 343:d3736. https://doi.org/10.1136/bmj.d3736

22. Care Quality Commission (2018) Mental Health Act - The rise in the use of the MHA to detain people in England. www.cqc.org. uk/publications/themed-work/mental-health-act-rise-mha-detainengland

23. Herbert A, Wijlaars L, Zylbersztejn A et al (2017) Data resource profile: hospital episode statistics admitted patient care (HES APC). Int J Epidemiol 46:1093-1093i. https://doi.org/10.1093/ ije/dyx015

24. Benchimol EI, Smeeth L, Guttmann A et al (2015) The REporting of studies Conducted using Observational Routinely-collected health Data (RECORD) Statement. PLOS Med 12:e1001885. https://doi.org/10.1371/journal.pmed.1001885

25. Cameron AC, Trivedi PK (1990) Regression-based tests for overdispersion in the Poisson model. J Econom 46:347-364

26. Muggeo V (2003) Estimating regression models with unknown break-points. Stat Med 22:3055-3071. https://doi.org/10.1002/ sim. 1545

27. Muggeo V (2008) Segmented: an R package to fit regression models with broken-line relationships. R News 8:20-25

28. Muggeo V (2016) Testing with a nuisance parameter present only under the alternative: a score-based approach with application to segmented modelling. J Stat Comput Simul 86:3059-3067. https://doi.org/10.1080/00949655.2016.1149855

29. Clegg LX, Hankey BF, Tiwari R et al (2009) Estimating average annual per cent change in trend analysis. Stat Med 28:3670-3682. https://doi.org/10.1002/sim.3733

30. McNicoll A (2015) Mental health trust funding down $8 \%$ from 2010 despite coalition's drive for parity of esteem. In: Community Care. www.communitycare.co.uk/2015/03/20/mental-health-trustfunding-8-since-2010-despite-coalitions-drive-parity-esteem/

31. Green BH, Griffiths EC (2014) Hospital admission and community treatment of mental disorders in England from 1998 to 2012. Gen Hosp Psychiatry 36:442-448. https://doi.org/10.1016/j.genho sppsych.2014.02.006

32. Glover G, Arts G, Babu KS (2006) Crisis resolution/home treatment teams and psychiatric admission rates in England. Br J Psychiatry 189:441-445. https://doi.org/10.1192/bjp.bp.105.020362

33. Alzheimer's Research UK (2018) Dementia Statistics Hub - Hospitals. https://www.dementiastatistics.org/statistics/hospitals/. Accessed 1 Nov 2021

34. Harwood R, PD KN, Edwards G (2010) Development of a specialist medical and mental health unit for older people in an acute general hospital. MedicalCrises in Older people Discussion Paper Series. Issue 5:2010

35. McNicoll A (2014) Bed shortage sees mentally unwell children sent hundreds of miles for care. In: Community Care. https:// www.communitycare.co.uk/2014/02/20/mentally-ill-child 
ren-sent-hundreds-miles-care-due-bed-shortage/. Accessed 1 Nov 2021

36. Owen J (2018) Children are still being forced to travel far for mental healthcare. BMJ. https://doi.org/10.1136/bmj.k3769

37. Wootton M, Brough J, Squire J et al (2014) What should general paediatric wards be doing to support young people with eating disorders? Archives of Disease in Childhood. BMJ Publishing Group Ltd, pp A23-A23

38. Sadler K, Vizard T, Ford T et al (2018) Mental health of children and young people in England, 2017. NHS Digital

39. Ahmadi-Abhari S, Guzman-Castillo M, Bandosz P et al (2017) Temporal trend in dementia incidence since 2002 and projections for prevalence in England and Wales to 2040: modelling study. BMJ 358:j2856. https://doi.org/10.1136/bmj.j2856

40. Appleby J (2012) Drinking nation: have we had enough? BMJ 344:e2634. https://doi.org/10.1136/bmj.e2634

41. Barr B, Taylor-Robinson D, Scott-Samuel A et al (2012) Suicides associated with the 2008-10 economic recession in England: time trend analysis. BMJ 345:e5142-e5142. https://doi.org/10.1136/ bmj.e5142

42. Cummins I (2018) The impact of austerity on mental health service provision: a UK perspective. Int J Environ Res Public Health 15:1145. https://doi.org/10.3390/ijerph15061145

43. Zivin K, Paczkowski M, Galea S (2011) Economic downturns and population mental health: research findings, gaps, challenges and priorities. Psychol Med 41:1343-1348. https://doi.org/10.1017/ S003329171000173X

44. Holmes EA, O'Connor RC, Perry VH et al (2020) Multidisciplinary research priorities for the COVID-19 pandemic: a call for action for mental health science. Lancet Psychiatry. https://doi. org/10.1016/S2215-0366(20)30168-1

45. Gunnell D, Kidger J, Elvidge H (2018) Adolescent mental health in crisis. BMJ 361:k2608. https://doi.org/10.1136/bmj.k2608

46. Ford T, Hamilton H, Meltzer H, Goodman R (2007) Child mental health is everybody's business: the prevalence of contact with public sector services by type of disorder among British school children in a three-year period. Child Adolesc Ment Health 12:1320. https://doi.org/10.1111/j.1475-3588.2006.00414.x

47. The Junior MARSIPAN Group (2012) Junior MARSIPAN: Management of really sick patients under 18 with anorexia nervosa. Royal College of Psychiatrists London

48. Byford S, Petkova H, Stuart R et al (2019) Alternative communitybased models of care for young people with anorexia nervosa: the CostED national surveillance study. Health Serv Deliv Res 7:1-78. https://doi.org/10.3310/hsdr07370

49. Walker S, Mackay E, Barnett P et al (2019) Clinical and social factors associated with increased risk for involuntary psychiatric hospitalisation: a systematic review, meta-analysis, and narrative synthesis. Lancet Psychiatry 6:1039-1053. https://doi.org/10. 1016/S2215-0366(19)30406-7 